\title{
Operando Electrochemical TEM of Solid-State Energy Storage Materials Using a Probe-Based Biasing Holder
}

\author{
Nikhilendra Singh ${ }^{1}$, James Horwath ${ }^{2}$, Alexandre Foucher ${ }^{2}$, Timothy S. Arthur ${ }^{1}$, Julio A. Rodríguez \\ $\mathrm{Manzo}^{3}$, Daan Hein Alsem ${ }^{3 *}$ and Eric Stach ${ }^{2,3}$ \\ 1. Toyota Research Institute of North America, Ann Arbor, MI, USA \\ 2. Department of Materials Science and Engineering, University of Pennsylvania, Philadelphia, PA, USA \\ 3. Hummingbird Scientific, Lacey, WA, USA. \\ * Corresponding author: daan_alsem@hummingbirdscientific.com
}

With purpose-designed operando transmission electron microscope (TEM) biasing sample holders, it is possible to make very localized electrical contacts to a sample inside the TEM using a movable probe. If the sample and probe are biased at different electrical potentials, contacting the sample with the movable probe creates an electrical circuit (Figure 1). This operando TEM sample biasing platform allows for the investigation of electrical properties of small contacts and correlating this with their structural and chemical state, by using all imaging and spectroscopy techniques (high-resolution imaging, electron-based spectroscopy, electron diffraction, etc.) available in the TEM. This method has previously been used to investigate, the switching of oxide resistive memories [1], anode materials for batteries [2,3], and the conductance through rows of atoms [4,5].

Obtaining reliable, quantitative information from operando biasing experiments in the TEM that can be compared with "bulk" experiments can be challenging. Standards are lacking, and it is not uncommon to have contradictory results about the same sample from different experiments. In this context, here we concentrate on the practical aspects of creating very local electrical contacts to study energy storage materials for next generation solid-state batteries and conducting repeatable operando experiments inside the TEM. We will show experiments performed with the probe-based sample holder which allow for the approach of a sample with the movable probe - using a scanning tunneling microscopy (STM) tip-in manual coarse mode with smooth and uncoupled motion with probe alignment to below $1 \mu \mathrm{m}$ on the three axes of motion. After that, the TEM biasing holder can perform a final contact approach of the probe to the sample with sub-nm accuracy (Figure 1) with a piezoelectric controlled mechanism which permits uncoupled nanometer-sized (or less) steps in three dimensions.

We present an operando TEM study of solid-state electrolyte particles as an example of this technique (Figure 2). Micron-sized particles were contacted with a mobile probe and cyclic voltammograms were obtained. Currents in the order of picoamps were measured and, simultaneously, electrochemical induced structural changes, within particles and at interfaces, were imaged in the TEM. Our results show clear correlations between gathered electrochemical data and structural transformations. This approach is versatile and can be used to study any solid-state electrolyte/electrode configuration. A key aspect in acquiring this data outside of being able to make these local electrical connections was to keep the airsensitive sample surfaces "pristine", which was done by preparing and loading the sample into the TEM holder within a glove box, as well as using an air-free transfer cover to transfer the sample and TEM holder into the TEM. Protected sample preparation and transfer to the TEM provided repeatable results between experiments. The latter also illustrates that practical considerations can have a large impact on the outcome of the experiment and controlling these aspects is key to acquiring consistent data from operando microscopy experiments. 
References:

[1] David Cooper, et al., Advanced Materials 29 (2017), p. 1700212.

[2] Fei-Hu, et al., ACS Nano 11 (2017), p. 8628.

[3] Ze Yang, et al., Energy Storage Materials 9 (2017), p. 214

[4] Hideaki Ohnishi, Yukihito Kondo, Kunio Takayanagi. Nature 395 (1998), p. 780.

[5] Ovidiu Cretu, et al., Nano Letters 13 (2013), p. 3487.
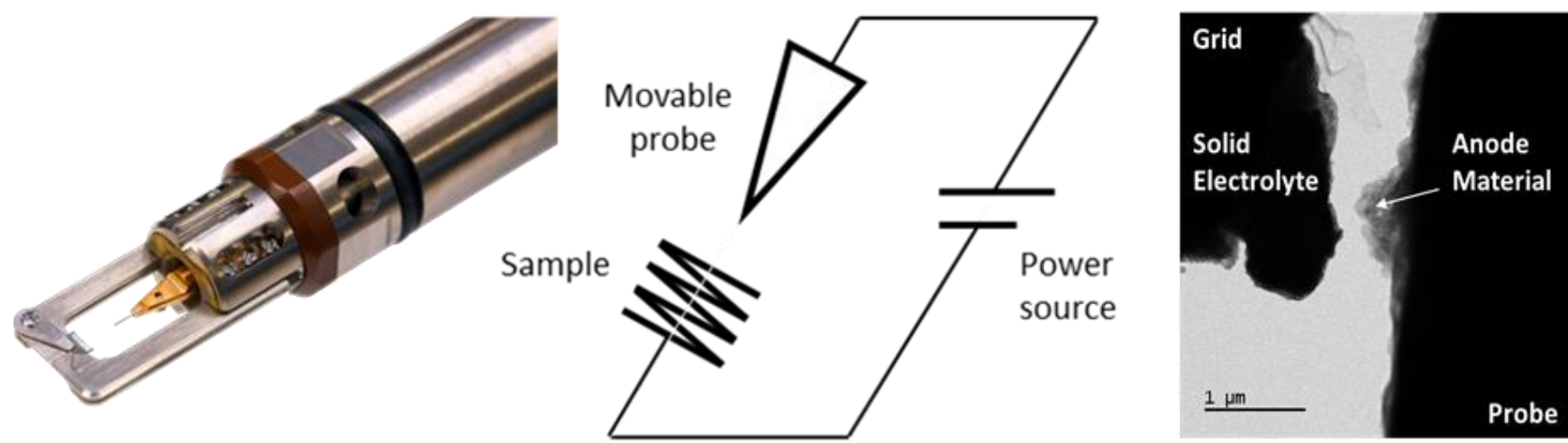

Figure 1. Details of the operando TEM biasing sample holder with a movable probe. From left to right, tip of the operando TEM biasing sample holder, detailed circuit diagram and a low magnification TEM image highlighting the holder geometry as observed in the TEM.
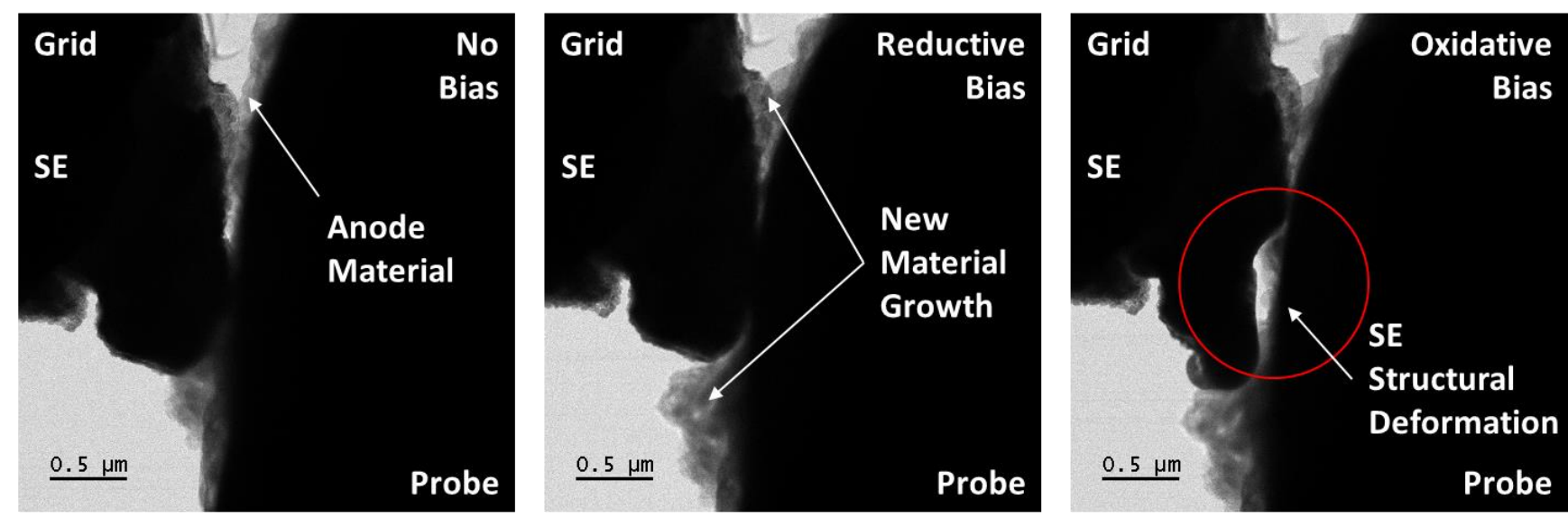

Figure 2. Operando TEM images taken for a combination of solid electrolyte (SE) and anode material in the biasing sample holder with a movable probe. From left to right, SE and anode material brought in contact without electrical biasing, SE/anode material under reductive bias at the probe and SE/anode material under oxidative bias at the probe highlighting structural deformation of the SE. 